\title{
The dynamic mechanical characteristics of a resonating microbridge mass-flow sensor
}

\author{
H. J. M. Geijselaers and H. Tijdeman \\ Applied Mechanics, Mechanical Engineering, University of Twente, P.O. Box 217, 7500 AE Enschede (The Netherlands)
}

(Received December 11, 1990; in revised form February 26, 1991; accepted March 19, 1991)

\begin{abstract}
This paper gives an explanation of the dynamic mechanical behaviour of a resonating microbridge mass-flow sensor. A rise in the average temperature of the bridge initially results in a reduction of the resonance frequency. Upon further temperature rise, a reversal occurs and the resonance frequency starts rising too. The dynamic behaviour in this case is found to be governed by static buckling. This phenomenon is analysed, first using a finite-element model and then with an approximate analytical calculation.
\end{abstract}

\section{Introduction}

The technology of micromachining of silicon crystals provides challenging opportunities for the development of micromechanical structures [1]. The class of resonating silicon sensors [2], which have high sensitivity, high resolution, low power consumption and low drift, is very promising. Their application as mass-flow sensors is being studied at the University of Twente [3].

The mass-flow sensor considered here (Fig. 1) consists of a silicon nitride microbridge with embedded polysilicon resistors, sus-

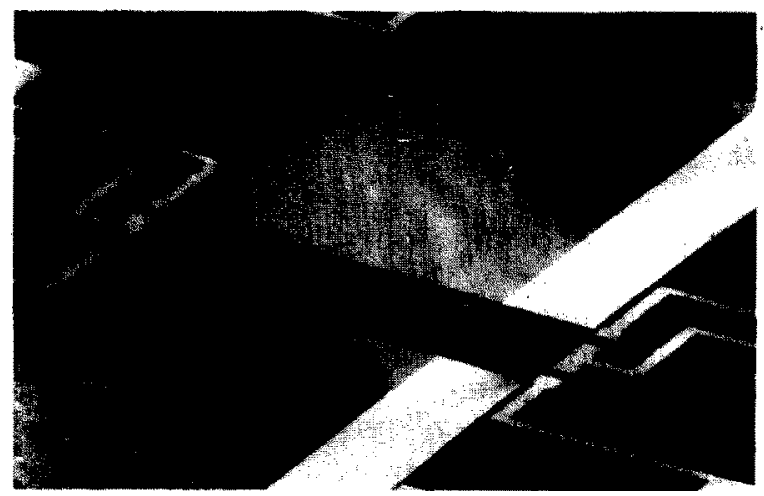

Fig. 1. SEM photograph of an uncapped resonating microbridge mass-flow sensor. Dimensions: $600 \mu \mathrm{m} \times 200$ $\mu \mathrm{m} \times 2.1 \mu \mathrm{m}[3]$. pended inside a micro flow channel. The thinfilm process conditions are such that the silicon nitride film exhibits a small residual tensile stress.

The bridge is forced into vibration by thermal excitation. This also causes a static average temperature elevation of the microbridge. Convective heat transfer to the passing flow results in a drop of the average equilibrium temperature of the microbridge. A change in temperature causes thermal dilatation and influences the axial force in the bridge. This results in a change of the resonance frequency of the bridge.

By monitoring the shift in resonance frequency, the mass flow through the micro flow channel can be determined. A rise in the average temperature of the bridge initially results in a reduction of the resonance frequency. Upon further temperature rise, a reversal occurs and the resonance frequency starts rising too $[3,4]$.

\section{Finite-element analysis}

A finite-element model is constructed, based on the actual dimensions and material data (Table 1) of the microbridge, using 16 eight-node shell elements. The ANSYS finiteelement program is used. In this model the 
TABLE 1. Material properties of the silicon nitride microbridge

\begin{tabular}{lll}
\hline Young's modulus: & $E=225$ & $\mathrm{GPa}$ \\
Poisson ratio: & $\nu=0.3$ & \\
Thermal expansion coefficient: & $\alpha=2.5 \times 10^{-6}$ & $\mathrm{~K}^{-1}$ \\
Initial axial stress: & $\tau_{0}=26$ & $\mathrm{MPa}$ \\
Mass density: & $\rho=3000$ & $\mathrm{~kg} \mathrm{~m}$ \\
\hline
\end{tabular}

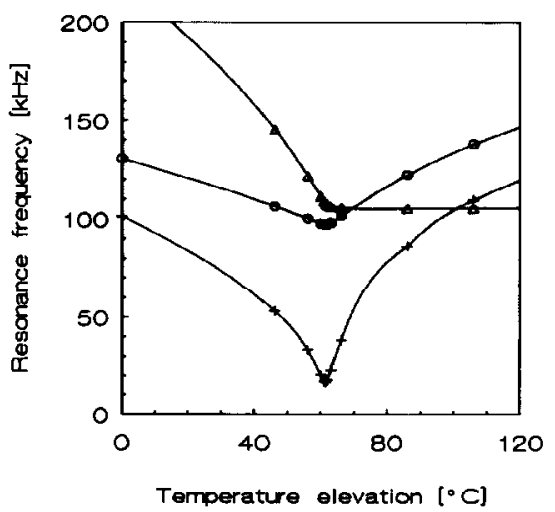

Fig. 2. Variation of resonance frequencies with temperature rise, finite-element results. + , first bending; $O$, first torsion; $\triangle$, second bending.

bridge is not straight, but has an initial deflection with a value of $0.02 \mu \mathrm{m}$ at the centre of the bridge. This initial deflection, which corresponds to approximately $1 \%$ of the thickness of the bridge, is necessary to enable non-linear deflection calculations up to and beyond the buckling temperature.

The structural boundary conditions of the bridge are such that all displacements and rotations are suppressed where it is supported. In successive steps the temperature is raised and at each step a new non-linear static equilibrium deflection is calculated. Next the resonance frequencies of vibrations about these deflected positions are calculated.

The variation of the first three resonance frequencies with temperature is shown in Fig. 2. The corresponding vibration modes at two different values of the temperature elevation are depicted in Fig. 3.

Due to the rise in temperature, the initial tensile stress in the bridge is lowered. As a result, the stiffening effect of the tensile stress decreases, which accounts for the initial drop in the values of the resonance frequencies that can be observed for a temperature elevation lower than $60^{\circ} \mathrm{C}$.

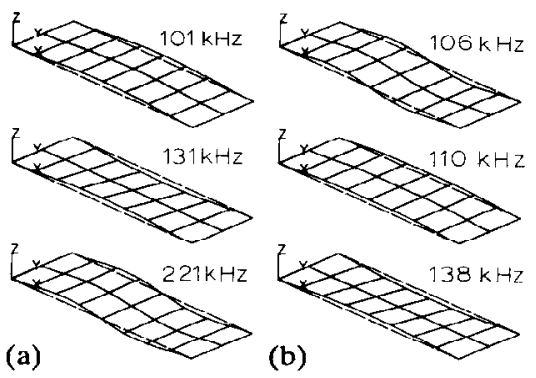

Fig. 3. Shapes of the first three vibration modes. (a) Temperature rise $=0{ }^{\circ} \mathrm{C}$; (b) temperature rise $=106^{\circ} \mathrm{C}$.

Beyond a temperature rise of approximately $45^{\circ} \mathrm{C}$ a compressive stress builds up. At a temperature elevation of about $60^{\circ} \mathrm{C}$ the compression equals the buckling stress of the bridge. Beyond this temperature, the compressive stress does not rise any more. The increase in thermal expansion causcs a static transverse deflection in the form of the buckling mode.

As a result the initially (almost) straight beam becomes curved, which gives rise to a coupling between transverse deflection and axial strain. This coupling results in an increase in resonance frequency with temperature rise, which is most pronounced for the first bending mode, but is also considerable for the first torsion mode. The second bending mode is (for reasons to be explained below) not affected at all.

Beyond a temperature rise of about 100 ${ }^{\circ} \mathrm{C}$, a jump-over occurs and the second bending mode becomes the fundamental resonance mode, as can be observed from Fig. 2 and from the sequence of the mode shapes in Fig. 3.

\section{Approximate analytical model}

Here we confine ourselves to an approximate analysis with an explanation of the physical characteristics of the problem. For a more thorough treatment of the dynamics of slightly curved beams under axial compression, the reader is referred to Kim and Dickinson [5]. We concentrate on the first and second bending resonance modes. The first torsion mode may be treated in a similar but slightly more involved way. 
(a) Approximation of vibration modes

The model of the microbridge is that of a straight beam. A straight, unstressed beam, clamped at both sides, resonates in transverse deflection modes whose shapes are given by

$$
\begin{aligned}
w_{i}(x)= & C_{1} \sin \left(k_{i} x\right)+C_{2} \cos \left(k_{i} x\right) \\
& +C_{3} \sinh \left(k_{i} x\right)+C_{4} \cosh \left(k_{i} x\right)
\end{aligned}
$$

where $x$ is the coordinate along the beam and $w_{i}(x)$ is the transverse deflection. $C_{j}$ and $k_{i}$ are found by substituting the appropriate boundary conditions and solving the resulting eigenvalue problem.

However, in view of the approximate nature of the present calculations and for ease of evaluating the resulting integrals, the first and second bending modes are approximated by

$w_{1}(x)=W_{1}(1-\cos (2 \pi x / L))$

and

$$
\begin{aligned}
w_{2}(x)= & W_{2}(1-2 x / L-\cos (2.86 \pi x / L) \\
& +0.223 \sin (2.86 \pi x / L)),
\end{aligned}
$$

respcctively, where $x$ is the coordinate along the beam, $L$ is the beam length and $W_{1}$ and $W_{2}$ are amplitude parameters.

(b) Behaviour below the buckling temperature

Raising the temperature of the beam while keeping the length between the supports constant causes an axial force $N_{\mathrm{T}}$ in the beam:

$N_{\mathrm{T}}=N_{0}-S_{\mathrm{c}} \alpha \Delta T$

where $N_{0}$ is the force in the beam at zero temperature rise, $S_{\mathrm{e}}$ is the extensional stiffness of the beam, $\alpha$ is the coefficient of thermal expansion and $\Delta T$ is the temperature rise. A positive value of $N_{\mathrm{T}}$ corresponds to tension. The influence of the axial force on the resonance frequencies can be estimated by using Rayleigh's quotient [6] as explained below.

It is reasonable to assume that the shapes of the resonance modes change only negligibly with increasing axial force. The expression for the deformation energy is

$$
\begin{aligned}
P\left(W_{i}, \Delta T\right)= & \frac{1}{2} \int\left\{S_{\mathrm{b}}\left(w_{i, \mathrm{x} x}\right)^{2}\right. \\
& \left.+N_{\mathrm{T}}\left(w_{i, x}\right)^{2}\right\} \mathrm{d} x \quad i=1,2
\end{aligned}
$$

$S_{\mathrm{b}}$ is the bending stiffness of the beam ( $)_{2 x}$ and ()$_{x x}$ denote the first and second derivatives with respect to $x$.
The kinetic energy is found as

$T\left(W_{i}\right)=\frac{1}{2} 4 \pi^{2} \nu_{i}^{2} \int m w_{i}^{2} \mathrm{~d} x \quad i=1,2$

where $\nu$ is the resonance frequency and $m$ is the weight per unit length of the beam. The approximate values of the resonance frequencies of the first and second bending modes are then found as

$$
\begin{gathered}
\nu_{i}(\Delta T)=\frac{1}{2 \pi}\left[\int\left\{S_{\mathrm{b}}\left(w_{i, x x}\right)^{2}+N_{\mathrm{T}}\left(w_{i, x}\right)^{2}\right\} \mathrm{d} x\right. \\
\left./ \int m w_{i}^{2} \mathrm{~d} x\right]^{1 / 2} \quad i=1,2
\end{gathered}
$$

The fundamental resonance frequency $\nu_{1}$ becomes zero when the value of the compressive force reaches the buckling value:

$N_{\mathrm{c}}=-4 \pi^{2} S_{\mathrm{b}} / L^{2}$

which corresponds to a critical temperature elevation

$$
\Delta T_{\mathrm{c}}=\left(N_{\mathrm{o}}+4 \pi^{2} S_{\mathrm{b}} / L^{2}\right) / \alpha S_{\mathrm{e}}
$$

(c) Behaviour above the buckling temperature

A further rise of the temperature causes no rise of the compressive force, but the initially straight beam adopts a curved shape $z(x, \Delta T)$, which is approximately characterized by the buckling mode:

$$
z(x, \Delta T)=A[1-\cos (2 \pi x / L)]
$$

The maximum deflection is $2 A$ at the centre, where $A(\Delta T)$ is obtained by calculating the length of the deflected shape:

$A(\Delta T)=(L / \pi)\left[\alpha \Delta T+\left(N_{\mathrm{c}}-N_{\mathrm{o}}\right) / S_{\mathrm{e}}\right]^{1 / 2}$

When the beam performs vibrations about this deflected shape, the behaviours of the fundamental vibration mode and of the second bending mode are quite different. We still assume that the (transverse) shapes of the resonance modes change only negligibly.

Superposition of displacements $\{u(x), w(x)\}$, where $u$ is the axial displacement component, upon the curved shape of the beam causes not only bending, but also stretching $\epsilon$ given by

$$
\epsilon(x)=u_{, x}+z_{, x} w_{, x}
$$


(i) First bending mode

The axial displacements $u_{1}(x)$ of the fundamental mode are very small (Fig. 4(a)), so the stretch $\epsilon$ is approximated by

$\epsilon \cong z_{, x} W_{1, x}$

This gives rise to an additional term in the deformation energy:

$$
\begin{aligned}
P\left(W_{1}, \Delta T\right)= & \frac{1}{2} \int\left\{S_{\mathrm{b}}\left(w_{1, x x}\right)^{2}+N_{\mathrm{c}} w_{1, x}\right)^{2} \\
& \left.+S_{\mathrm{c}}\left(z_{, x}\right)^{2}\left(w_{1, x}\right)^{2}\right\} \mathrm{d} x
\end{aligned}
$$

The kinetic energy change is negligible. Due to the extra deformation energy, the resonance frequency $\nu_{1}$ shows a sudden steep rise when the temperature exceeds the buckling temperature $\Delta T_{\mathrm{c}}$.

\section{(ii) Second bending mode}

The second bending mode is not symmetric with respect to the centre of the beam. By adding small displacements $u_{2}(x)$ in the axial direction to the transverse displacements $w_{2}(x)$, extensionless bending is still possible (see Fig. 4(b)). From the condition of zero extension

$\epsilon=u_{2, x}+z_{, x} w_{2, x}=0$

it can be shown that the amplitude of the axial component is only of the order $(A / L) W_{2}$. The influence of $u_{2}$ on the bending can be shown to be even of the order $(A / L)^{2}$. Since $A / L \ll 1$, for temperatures higher than $\Delta T_{c}$, neither the deformation energy nor the kinetic energy changes, so the value of the second bending resonance frequency remains practically constant.

\section{(d) Comparison with measurements and} finite-element results

The frequencies obtained with the expressions derived here are compared to the finiteelement results and measured resonance frequencies in Fig. 5. For the microbridge considered here, only the fundamental resonance frequency was measured [4].

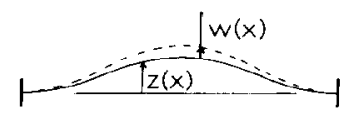

(a)

Fig. 4. Modes of vibration about a deflected shape $z(x)$.

(a) First bending mode; (b) second bending mode.

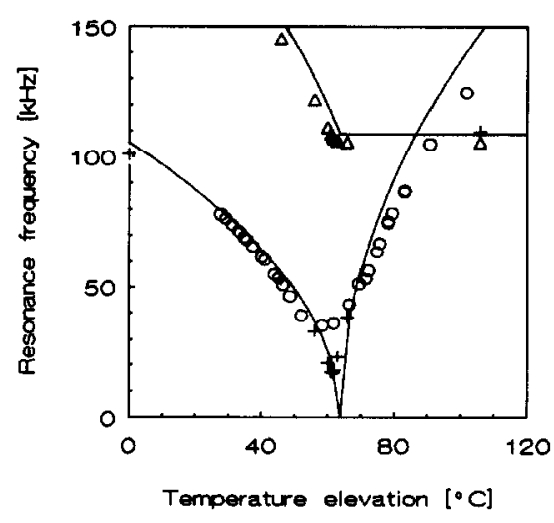

Fig. 5. Comparison of measured and calculated resonance frequencies. Analytical: - - first bending; - - second bending. Finite elements: + , first bending; $\Delta$, second bending. Measured: $O$, first bending [4].

The correspondence is excellent for values of temperature elevation $\Delta T$ up to $80^{\circ} \mathrm{C}$. At higher temperatures, the mode shape $w_{1}$ starts to change. This change is the reason for the discrepancies in the analytically determined $\nu_{1}$ at higher temperature elevations. A better correspondence at higher temperatures is obtained by using a two-term Rayleigh-Ritz approximation for the first vibration mode, as shown by Kim and Dickinson [5].

\section{Discussion}

The fact that the lowest frequency of the microbridge is not single valued with respect to the temperature represents a complication and restricts the range of the sensor. The higher gradient of the frequency curve at temperatures above the buckling temperature can be exploited to give enhanced sensitivity. A suitably optimized geometric design of the bridge may broaden the measurement range of the sensor, either by raising the buckling temperature, if measurement below this temperature is desired, or raising the jump-over temperature, if measurement is to be done at temperatures above the buckling temperature.

In order to prevent the jump-over between the first and second bending modes, the layout of the thermal excitation resistor circuit must be such that only symmetric vibration modes are excited. 


\section{Acknowledgements}

The authors like to thank Siebe Bouwstra and Rob Legtenberg of the Research Unit Sensors and Actuators of the University of Twente for putting their experimental results at our disposal and for their useful comments.

\section{References}

1 K. E. Petersen, Silicon as a mechanical material, Proc. IEEE, 70 (5) (1982) 420-457.

2 R. M. Langdon, Resonator sensors, a review, J. Phys. E: Sci. Instrum., 18 (1985) 103-115.

3 S. Bouwstra, Resonating micro bridge mass flow sensor, Ph.D. Thesis, University of Twente, Enschede, The Netherlands, 1990.

4 R. Legtenberg, S. Bouwstra and J. H. J. Fluitman, Resonating microbridge mass-flow sensor with lowtemperature glass bonded cap wafer, Sensors and ACtuators $A$, 25-27 (1991) 723-727.

5 C. S. Kim and S. M. Dickinson, The flexural vibration of slightly curved slender beams subject to axial end displacement, J. Sound Vib., 104 (1) (1986) 170-175.
6 L. Meirovitch, Elements of Vibration Analysis, McGrawHill, New York, 2nd edn., 1986, Ch. 7.

\section{Biographies}

Hubertus J. M. Geijselaers obtained an M.Sc. in aeronautical engineering in 1980 from Delft. From 1981 to 1985 he worked for Fokker Space and Systems.

Since November 1987 he has been a lecturer at the applied mechanics section of the faculty of mechanical engineering of the University of Twente. His main field of research is thermo-mechanical forming processes.

Hendrik Tijdeman obtained an M.Sc. from Delft in 1961 and a Ph.D. from Delft in 1977, both in aeronautical engineering. From 1961 to 1986 he worked at the National Aerospace Laboratory, NLR.

Since September 1986 he has been a professor of applied mechanics at the faculty of mechanical engineering of the University of Twente. His main field of research is structural dynamics. 